\title{
EL DESARROLLO DE ARMADURAS PARA TENDELES A LO LARGO DE DOS DÉCADAS
}

\section{(THE DEVELOPMENT OF BED JOINT REINFORCEMENT OVER TWO DECADES)}

\author{
Oscar Pfefferman, Profesor Dr. Ingeniero \\ STUDIEBUREAU B.T.C. Amberes. \\ BÉLGICA
}

Barry A. Haseltine, Dr. Ingeniero

Presidente del Eurocódigo SC6 "Estructuras de fábrica".

JENKINS \& POTTER Consulting Engineers, Londres.

REINO UNIDO

Fecha de recepción: 8-IX-92

\section{RESUMEN}

En Europa, la experiencia acumulada a lo largo de 20 años de investigación y desarrollo con las armaduras prefabricadas planas (Murfor), permite fijar las especificaciones de calidad que han de cumplimentar las armaduras para la fábrica armada, de acuerdo a la normativa europea en desarrollo sobre Estructuras de Fábrica Armada (EC6).

En el artículo se analizan los tipos de armaduras con sus campos de aplicación, así como los ensayos a que son sometidas para garantizar su buen comportamiento y evitar la corrosión.

Se complementa con una investigación que muestra las mejoras obtenidas en la edificación al utilizar la fábrica armada.
SUMMARY

The experience accumulated over twenty years of research and development with prefabricated reinforcement for masonry (Murfor), allows to establish the specifications of quality which have to be fulfilled by the reinforcement for the masonry in accordance with the European guidelines for development of Reinforced Masonry Structures (EC6).

This article analyzes the types of masonries reinforcement with their application fields as well as the tests they are put to, in order to guarantee their satisfactory behaviour and avoid corrosion.

$A$ research which shows the improvements obtained in building by using the reinforced masonry is also described in the article.

\section{INTRODUCCIÓN}

En el período de 1964 a 1966, el "Centre Scientifique et Technique de la Construction" hizo una inspección sobre las obras realizadas, a fin de establecer las causas de deterioro en la construcción. Esta inspección, llevada a cabo con técnicos, arquitectos, ingenieros, constructores y también con laboratorios y promotores de viviendas y edificios, mostró que el agrietamiento en la albañilería era con mucho el daño más observado (más del $40 \%$ de los casos analizados).
Diferentes causas llevaban a este agrietamiento:

- Causas de origen físico: empuje de cubierta o movimientos de la estructura de hormigón armado, contracción y dilatación de paredes...

- Causas de origen mecánico: excesiva carga en muros, descensos de forjados o vigas de apoyo de tabiques...

- Causas accidentales: asentamiento de cimientos, vibraciones... 


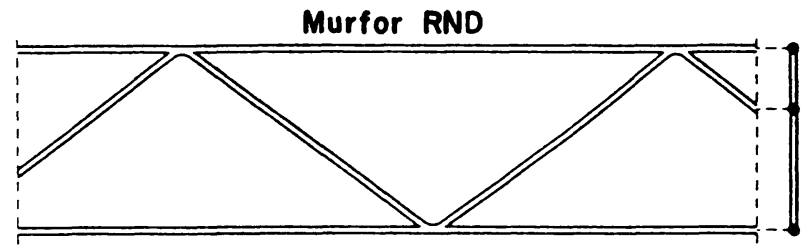

COMPUESTA POR ALAMBRES, PARA LAS OBRAS DE FÁBRICA CON JUNTAS DE MORTERO NORMALES.

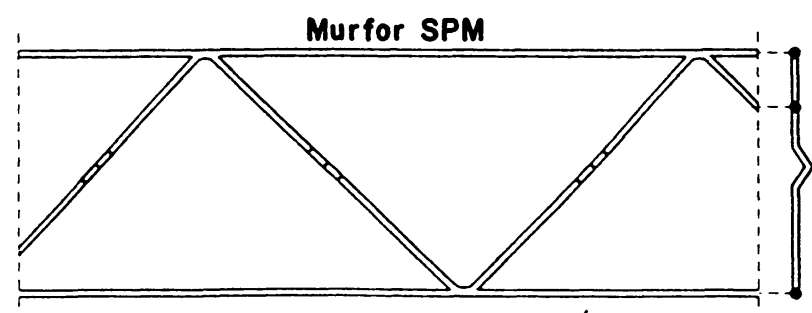

COMPUESTA POR ALAMBRES, CON UN DOBLÉZ PARA MUROS CAPUCHINOS, EN MUROS DOBLES.

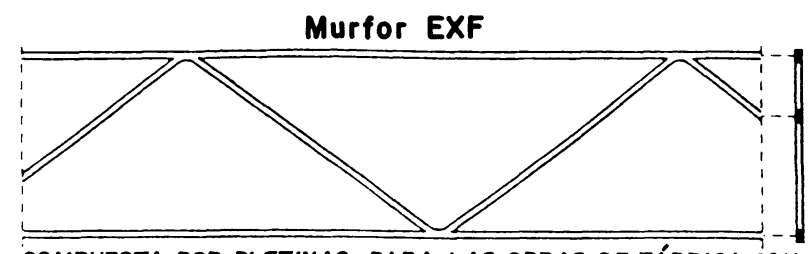

COMPUESTA POR PLETINAS, PARA LAS OBRAS DE FÁBRICA CON JUNTAS FINAS DE MORTERO COLA.

Fig. 1.-Descripción de los tipos de armaduras estándar.

En todos estos ejemplos la limitada resistencia a tracción y a corte de la albañilería han sido puestos en evidencia, de aquí la idea de reforzar la albañilería de modo exactamente análogo al armado del hormigón para incrementar su capacidad de soportar cargas.

De allí viene la idea de MURFOR, un refuerzo especialmente adaptado a la albañilería. Para determinar sus características y su efectividad se ha desarrollado un enorme programa de ensayos en un momento en el que la fábrica armada era casi desconocida en Europa continental.

\section{ARMADURA MURFOR}

La figura 1 muestra este tipo de armadura MURFOR. Se prefabrica en diversas anchuras y revestimientos, de acuerdo con el tipo de albañilería que debe ser construida.

En esta figura se pueden observar 3 formas:

1. Alambre redondo para fábricas con juntas de mortero normales.

2. Alambre redondo con un doblez en la retícula para muros capuchinos o doblados.
3. Alambre plano o pletinas para albañilería con tendeles finos.

También se pueden ver 3 revestimientos diferentes:

1. Alambre plano galvanizado por inmersión para albañilería expuesta a un entorno seco.

2. Revestimiento epoxi, al menos 100 micras en alambre galvanizado para albañilería expuesta a un entorno húmedo.

3. Acero inoxidable, para albañilería expuesta a un medio agresivo.

La primera aplicación con paredes de bloque de hormigón aligerado mostró que MURFOR era una excelente solución para evitar grietas. Algunos de los casos más significativos son:

- Largos paños de muro entre juntas de dilatación. La contracción y la dilatación limitan la longitud que los paños de pared pueden tener entre juntas de dilatación. El armado para tendeles controla la fisuración, haciendo posible un aumento de esta longitud entre un 50 y $100 \%$ (fig. 2).

- Muros o hastiales sometidos al empuje de cubiertas o a los movimientos de la estructura de hormigón.

- Muros de carga sobre vigas con peligro de flexión.

- Muros divisorios sobre forjados sujetos a deformación. En este último caso es habitual la fisuración de la tabiquería; se evita armando una serie de tendeles en la parte inferior de la pared, y dejando algún espacio entre la fábrica y el forjado superior o viga que permita su flexión.

Pero, lentamente, se han desarrollado otros campos estructurales distintos para el uso de MURFOR.

La figura 2 presenta algunas de estas aplicaciones comunes:

- La colocación de armaduras en los 3 ó 5 primeros tendeles impide la formación de grietas debidas a un eventual asentamiento diferencial del terreno.

- Concentración de tensiones alrededor de los huecos de puertas y ventanas, una causa conocida de fisuración que se neutraliza con la incorporación de armaduras en los tendeles por encima y por debajo de estas aperturas. 


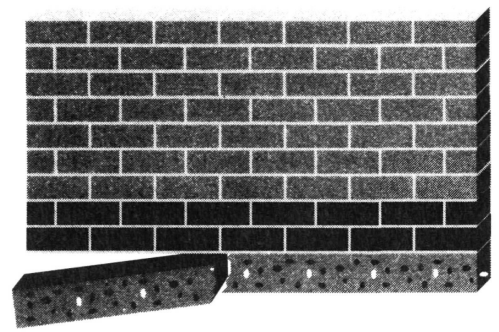

Riesgos de asientos.

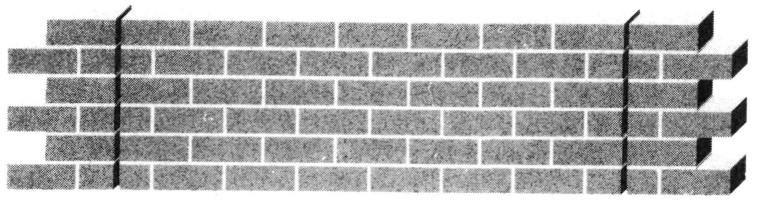

Largos paños de muro entre las juntas de dilatación.

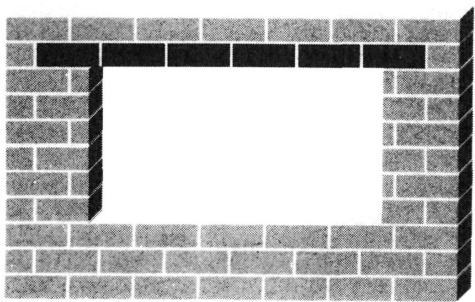

Los dinteles en la obra de fábrica.

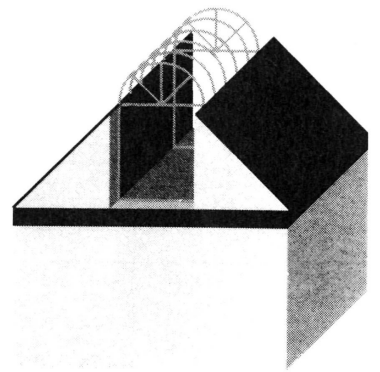

Cadenas de enlace y reparto.

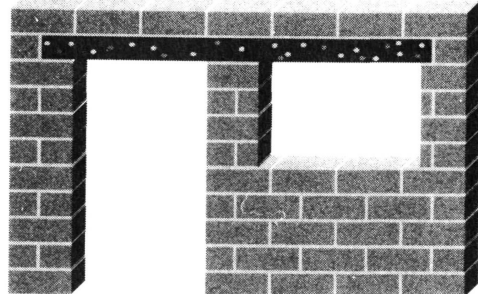

Concentración de tensiones alrededor de los huecos de puertas y ventanas.

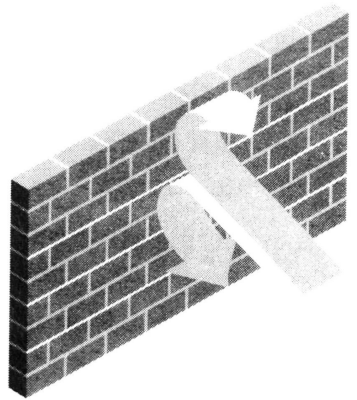

Grandes superficies de muro sujetas a la presión del viento

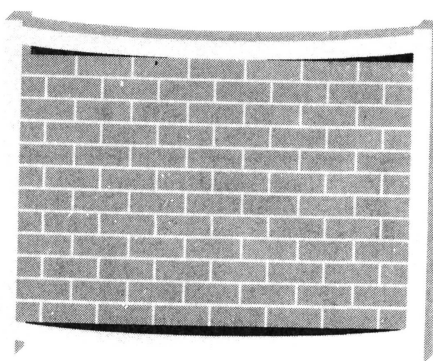

Muros sobre forjados o vigas sometidas a flexión

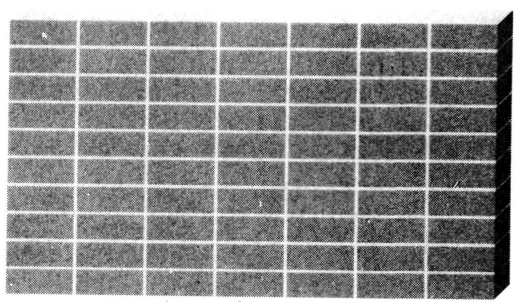

Fábrica a cara vista sin aparejar.

Fig. 2.-Aplicaciones de las armaduras Murfor. 
- En nuestros países las acciones del viento son asuntos serios, y todo diseñador ha de cumplir con severas especificaciones en las cargas de cálculo. Armar los tendeles de la fábrica es la mejor alternativa para evitar tener que construir muros gruesos entre soportes verticales.

- La obra de fábrica vista es afectada visual y físicamente por la incorporación de dinteles hechos de materiales ajenos (por ej. hormigón o acero). Los dinteles de fábrica con tendeles armados han llegado a ser ahora una buena manera de resolver este problema.

- Las cadenas de enlace y reparto se necesitan para rigidizar las paredes y para conectarlas. Para ejecutarlas en hormigón se necesitan encofrados caros, que requieren tiempo y perturban la homogeneidad física de la albañilería. Las armaduras en los tendeles hacen perfectamente esta función.

- Los arquitectos a los que les gusta la fábrica vista valoran las cualidades estéticas de este maravilloso material. La armadura para tendeles les ofrece a menudo nuevas posibilidades: las fábricas cara vista sin aparejar, incluso combinando diversos materiales, son ahora factibles utilizando armaduras prefabricadas en los tendeles.

\section{ESTUDIO EXPERIMENTAL}

En el período entre 1970 y 1980 se ha llevado a cabo, en Bélgica, un programa de investigación muy importante sobre fábrica armada. El programa de ensayos ha sido llevado por el CSTC ya mencionado, en colaboración con:

- La "Katholieke Universiteit Leuven" (KUL), que hizo ensayos mecánicos y de contracción.

- La "Rijksunivesiteit Gent (RUG)", para ensayos de fuego.

- La compañía Bakaert, para ensayos de corrosión in situ en Hemiksem, cerca de Amberes.

- La estación experimental del CSTC, en Limelette, para ensayos de corrosión acelerada.

2.1. Ensayos mecánicos de las fábricas (tabla 1)

2.1.1. Los materiales usados para ensayos incluyen:

- Bloque de hormigón, ladrillo cerámico, bloque de cerámica aligerada, bloque de hormigón celular.

- Morteros adaptados al material de fábrica de acuerdo con especificaciones belgas. Para el hormigón celular se usó una mezcla.

TABLA 1

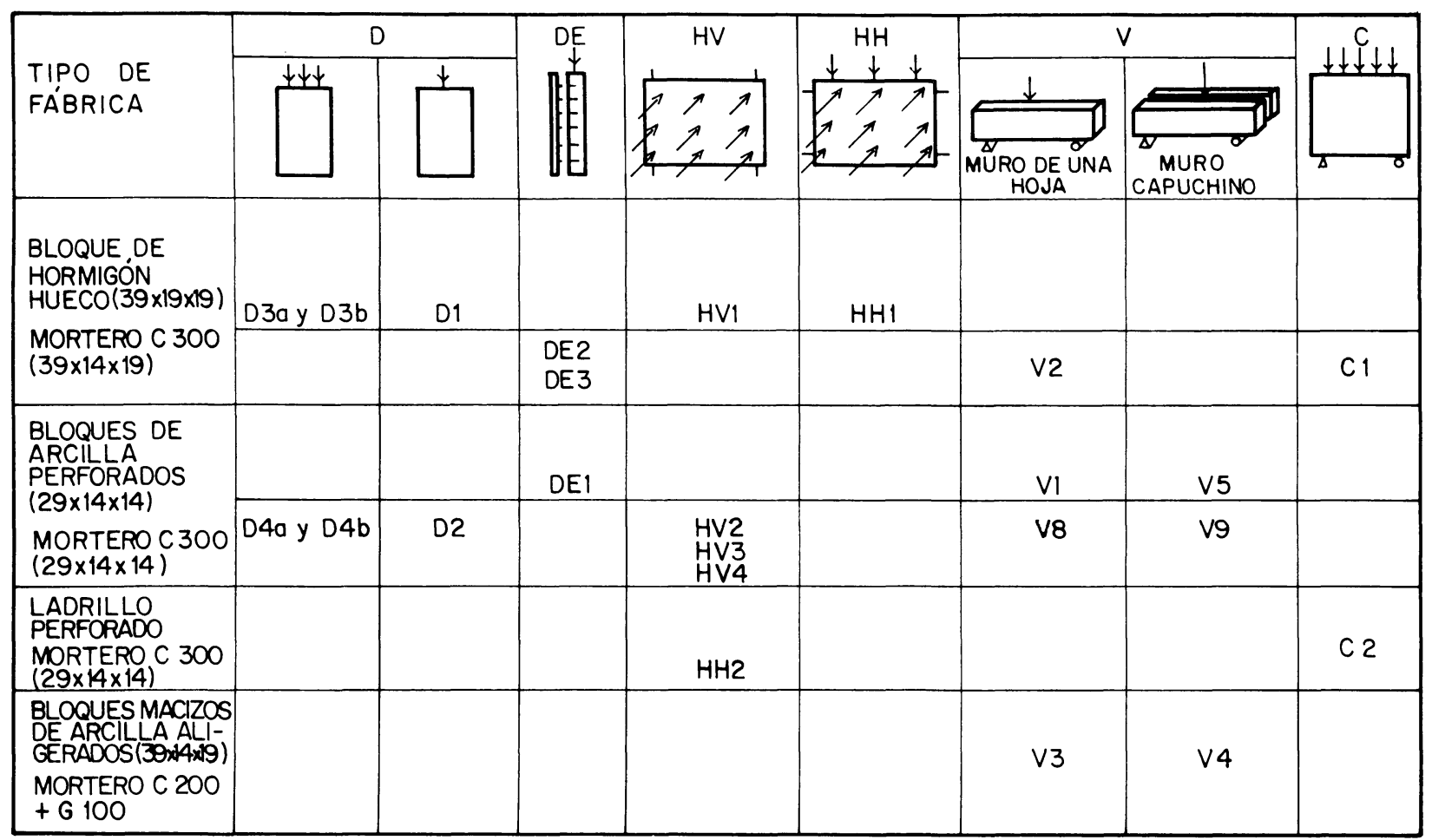


- Armaduras prefabricadas para tendeles tipo Murfor.

2.1.2. Los ensayos incluyen:

- Compresión concentrada y excéntrica.

- Flexión horizontal.

- Flexión vertical.

2.1.3. Resultados de ensayos y conclusiones:

- Compresión: La armadura absorbe tensiones de tracción longitudinales $\sigma_{1}$ y transversales $\sigma_{\mathfrak{t}}$, que aparecen en la albañilería bajo compresión debido al efecto Poisson. Por tanto, la fábrica armada ha aumentado su resistencia a la compresión de un 5 a un $20 \%$ en comparación con la de sin armar.

- Gracias al alambre central en zig-zag de la armadura se puede considerar conjuntamente el trabajo de las dos hojas de un muro capuchino. Un armado suplementario en ambas hojas absorbe el esfuerzo de corte. Este resultado positivo ha sido observado también con cargas excéntricas.

- Flexión: los ensayos de flexión horizontal y vertical han probado la validez de la hipótesis de la teoría de elasticidad; la correspondencia lineal de la deformación en función del momento se puso en evidencia.

\subsection{Ensayos físico-químicos del material}

2.2.1. Contracción: Se han sometido a ensayos cuatro paredes de hormigón celular de medidas $3,4 \times 1$ metro. La contracción se impidió confrontando las paredes contra un bastidor de acero y se le aplicó una carga vertical uniforme, a fin de simular la realidad. Sólo la pared no reforzada mostró formación de fisuras, las otras permanecieron sin fisurar.

2.2.2. Corrosión: Ensayos de corrosión acelerada de laboratorio. Se han expuesto (1,25 por 1,25 metros) a las siguientes condiciones: por un lado en condiciones de laboratorio normales $\left(20^{\circ} \mathrm{C}\right.$ y $60 \%$ de humedad relativa) y por otro lado, en una caja con condiciones extremas $\left(40^{\circ} \mathrm{C}\right.$ y $100 \%$ de humedad relativa). Después de ocho meses las paredes hechas con ladrillo cerámico hueco mostraron corrosión inicial en la armadura. Las paredes hechas de bloque de hormigón Besser no mostraron ninguna traza de corrosión en la armadura. En paredes dobles las diagonales de refuerzo que cruzan la cavidad se corroen si no se aplica ninguna capa de revestimiento protector adicional. Es necesario un recubrimiento mínimo de $15 \mathrm{~mm}$ de mortero bien rejuntado.

\section{MUROS EXTERIORES EXPUESTOS A LA CORROSION EN UN MEDIO INDUSTRIAL}

En 1976 se han construido en Hemiksem, cerca de Amberes, diferentes tipos de fábricas. Este área contiene una cantidad muy ligera de ácido clorhídrico, y puede ser considerada como un entorno industrial.

\subsection{Tipos de protección de la armadura}

- Galvanizado comercial por inmersión en caliente (min. $60 \mathrm{~g} / \mathrm{m}^{2}$ ).

- Galvanizado fuertemente por inmersión en caliente (mín. $275 \mathrm{~g} / \mathrm{m}^{2}$ ).

- Capa de galvalume (55\% Al, 40\% Zn, 5\% Si).

- Capa de epoxi sobre galvanizado (min. 80 micras epoxi sobre $60 \mathrm{~g} / \mathrm{m}^{2} \mathrm{Zn}$ ).

- Acero inoxidable (AISI 304).

\subsection{Tipos de muro (fig. 3)}

1) Ladrillo cerámico, $19 \mathrm{~cm}$ de ancho. Los ladrillos $(29 \times 19 \times 14 \mathrm{~cm})$ son del tipo "snelbouw" y están provistos de perforaciones verticales $(+/-15 \%)$. La calidad del mortero es C300 kg de cemento para $1 \mathrm{~m}^{3}$ de mortero. La dimensión de la pared es 1,40 m (altura) y 1,20 m (longitud).

2) Bloque de hormigón, $19 \mathrm{~cm}$ de ancho. Los bloques están provistos de dos huecos. La calidad del mortero es C400. Las dimensiones de las paredes son $1,40 \times 1,20$ metros.

3) Bloque çerámico aligerado, $19 \mathrm{~cm}$ de ancho. Los bloques $(39 \times 14 \times 19 \mathrm{~cm})$ son macizos. La calidad del mortero es C250 - G50 (200 kg de cemento y $50 \mathrm{~kg}$ de cal). Las dimensiones de las paredes son $1,40 \times 1,20$ metros. 


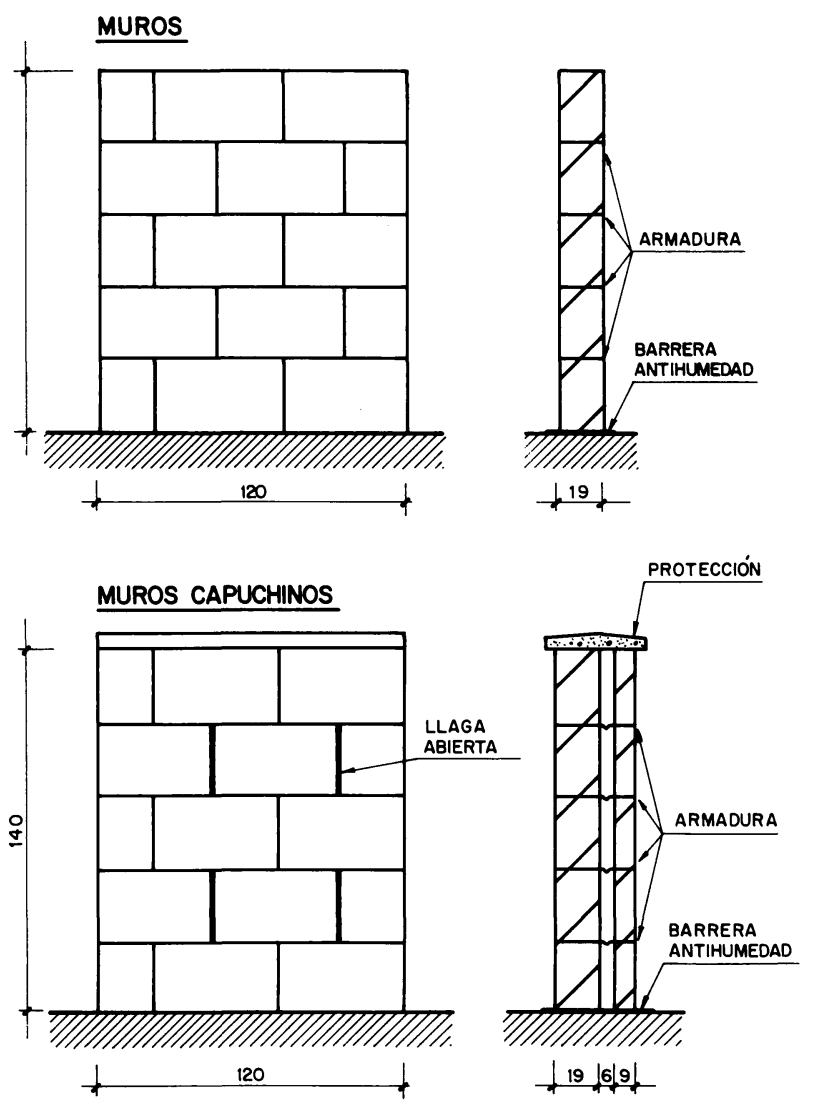

Fig. 3.-Tipos de muros ensayados.

4) Bloques de hormigón celular, $20 \mathrm{~cm}$ de ancho. Los bloques $(50 \times 24 \times 20 \mathrm{~cm})$ son macizos. El mortero contiene resinas sintéticas. El espesor de las juntas es $+/-2 \mathrm{~mm}$. Las dimensiones de la pared son $1,50 \times 1,20$ metros.

5) Muro capuchino de ladrillo cerámico. Dos paredes que tienen $9 \mathrm{~cm}$ de espesor, y la cavidad de $7 \mathrm{~cm}$. La calidad del mortero es C300. Las dimensiones de las paredes son $1,40 \times 1,20$ metros.

6) Muro capuchino de bloques de hormigón. Las paredes tienen un espesor de $9 \mathrm{~cm}$ (hoja externa) y $14 \mathrm{~cm}$ (hoja interna) respectivamente. La cavidad es de $7 \mathrm{~cm}$. El mortero tiene la calidad C400. Las dimensiones de las paredes son $1,40 \times 1,20$ metros.

\subsection{Resultados}

El análisis de las paredes demolidas condujo a una gran cantidad de importantes conclusiones, que influyeron en el desarrollo de las armaduras prefabricadas para los tendeles:
1) Alambres galvanizados comerciales

Hay alguna formación de óxido.

Se encontró una diferencia notable entre las paredes construidas con bloques de hormigón y las de ladrillo cerámico. En el caso de las paredes de ladrillos la corrosión es más grave y se detectó alguna oxidación local del acero. Para paredes de bloques de hormigón la corrosión está siempre uniformemente distribuida en toda la superficie.

El tipo plano de Murfor EXF, en combinación con bloques de hormigón celular y encolado con resinas sintéticas, muestra un buen comportamiento.

Las propiedades mecánicas:

La pérdida de resistencia a la rotura de los alambres llega a ser de 0 a $6 \%$ en los bloques de hormigón y hasta $15 \%$ en las paredes de ladrillo cerámico. La resistencia a la rotura del tipo plano EXF no ha cambiado. No se pudo detectar ninguna pérdida.

\section{2) El tipo fuertemente galvanizado en caliente}

Hay también formación de óxido. No se pudo encontrar ninguna diferencia con el tipo galvanizado normal.

Se detectó la misma diferencia entre ladrillos cerámicos y bloques de hormigón que en 1).

La corrosión está distribuida uniformemente sobre toda la superficie de los alambres en bloques de hormigón, mientras que en los ladrillos cerámicos se encontró alguna oxidación local más grave.

\section{Las propiedades mecánicas:}

Para paredes construidas con bloques de hormigón la pérdida en resistencia a la rotura era de 0 a 3\%, lo que no es significativo. Para paredes de bloques de cerámica la pérdida en resistencia a la rotura ascendía hasta el $12 \%$. El tipo plano EXF no mostró ninguna pérdida en resistencia a la rotura.

\section{3) El tipo galvalume}

El comportamiento de corrosión de este tipo es comparable con el tipo fuertemente galvanizado en caliente. La corrosión es aproximadamente 


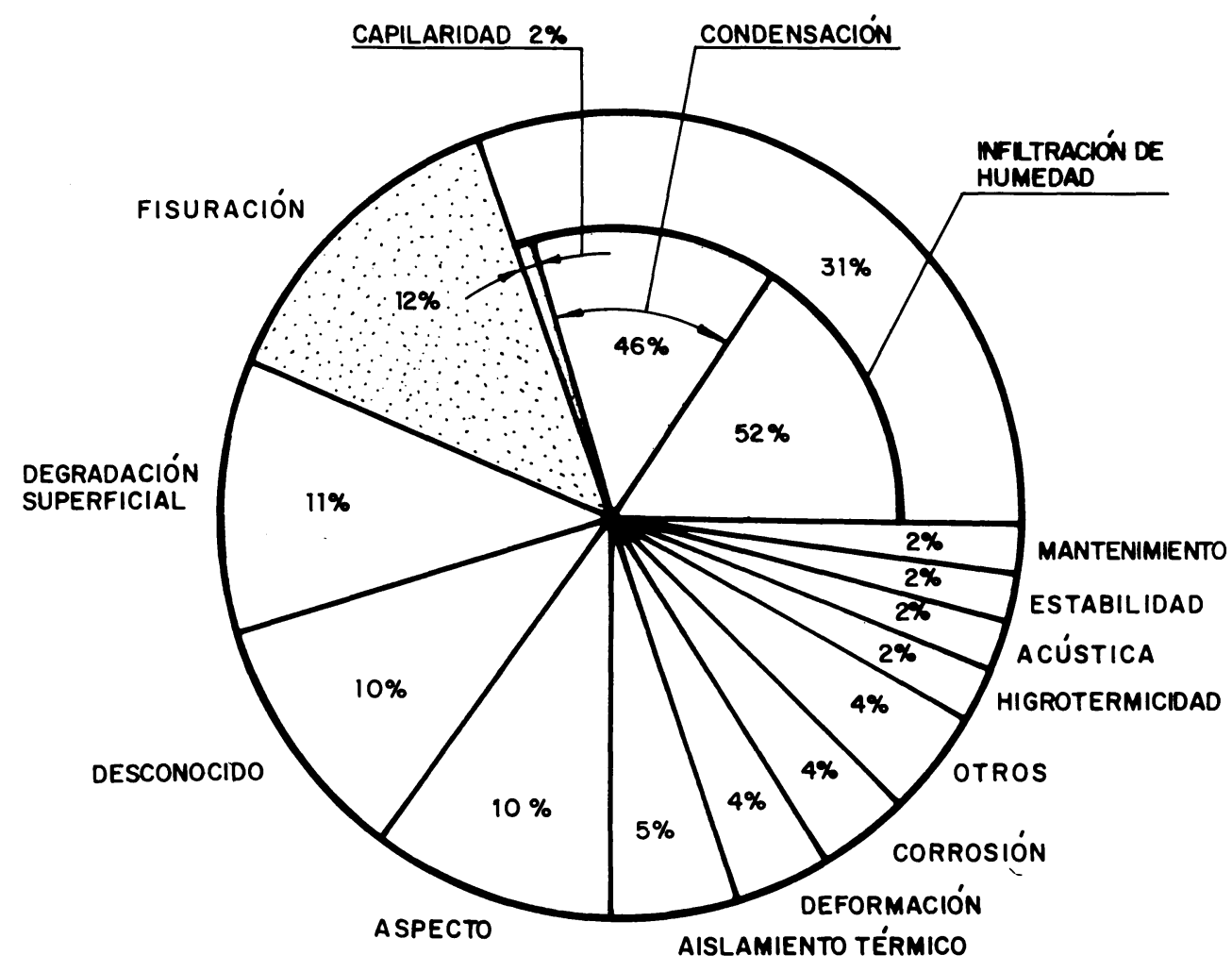

Fig. 4.-Diagrama del estudio sobre las causas de deterioro en las obras de fábrica. C.S.T.C. 1977-1979.

la misma en ladrillos cerámicos, con alguna oxidación local del acero. En bloques de hormigón el comportamiento está distribuido uniformemente sobre toda la superficie. El tipo plano EXF no se hizo en este tipo de recubrimiento.

Las propiedades mecánicas:

La pérdida de resistencia a la rotura no fue de más de $2,5 \%$ en bloques de hormigón, y hasta 9\% en las paredes de ladrillo cerámico.

4) El tipo galvanizado + revestido de epoxi

En todas las paredes este tipo salió totalmente intacto, sin corrosión ni revestimiento epoxi destruido.

Consiguientemente las propiedades mecánicas se encontraron intactas al $100 \%$.

5) El tipo de acero inoxidable

El comportamiento de corrosión de este tipo es $100 \%$ como era de esperar. No se pudo detectar ninguna oxidación y las propiedades mecánicas no han cambiado.

\subsection{Conclusiones generales}

1) Para paredes expuestas externamente y paredes en atmósferas corrosivas se necesita una protección especial.

No hay diferencia significativa entre galvanizado comercial, galvanizado fuerte en caliente y galvalume.

2) El tipo recubierto de epoxi es un material de refuerzo muy adecuado para construcciones con un tiempo de vida especificado muy largo.

3) El tipo de acero inoxidable, naturalmente, es un excelente material de armado pero no se comporta mejor en nuestros ensayos que los alambres recubiertos de epoxi.

\section{RESULTADOS PRÁCTICOS DE LA APLICACIÓN DE MURFOR}

Se mencionó, al principio de este artículo, la inspección sobre las obras construidas durante el año 1965 por el C.S.T.C. 
Una inspección similar fue ejecutada por el C.S.T.C. en los años 1977-1979. La figura 4 muestra la proporción del origen de los daños. Vemos que las fisuras representan generalmente el $12 \%$ del total de causas.

La subdivisión de esta cantidad del $12 \%$ se ha publicado por el C.S.T.C. (cómo tratar los defectos en la Construcción, N.o 6 Sept. 978) y se puede resumir como sigue:

- $20 \%$ de fisuras es causado por el asentamiento de cimientos;

- $34 \%$ de fisuras es producido por efectos térmicos;

- 16\% tiene su origen en variación de humedad;

- $30 \%$ de fisuras es producido por la deformación de los forjados o apoyos.

Se ha de subrayar, en la cantidad de fisuras, que no se está refiriendo sólo a la albañilería. Se está incluyendo también otros elementos como hormigón o elementos de acabado.
El mismo C.S.T.C. está llamando la atención, con regularidad, sobre las cuestiones técnicas generales planteadas por constructores o los casos inspeccionados por los técnicos del C.S.T.C.

Hay una constatación de que la cantidad de fisuras en las fábricas se ha estabilizado en un porcentaje menor del conjunto de daños existentes. Este serio decrecimiento del $40 \%$ al $12 \%$ de las grietas existentes en las fábricas, desde el año 65 al 78, no es debido solamente a la influencia exclusiva del uso de MURFOR.

Está combinada con una mejor selección de los elementos de construcción, aplicación de juntas de dilatación, etc.

Estamos seguros de que el uso de MURFOR, y los adecuados consejos dados por las consultorías técnicas en la fase de proyecto, llevan a una alta confianza en los materiales, y en la albañilería en general.

\section{publicación del ICCET/CSIC}

\section{bases para el diseño solar pasivo}

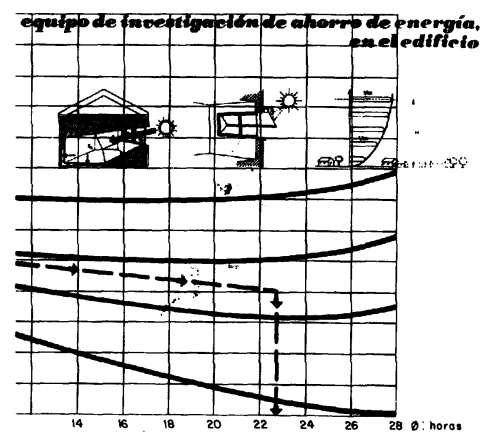

Institute Eduardo Torroje do In Construccion y del Cemento
CONSEJO SUPERIOR OE INVESTIGACIONES CIENTIFICAS
Equipo de Ahorro de Energía en el edificio

Dirección y coordinación:

Arturo Garcia Arroyo

M. a José Escorihuela

José Luis Esteban

José Miguel Frutos

Manuel Olaya

Bernardo Torroja
Las dificultades de suministro y el alto coste de los productos energéticos convencionales han despertado la atención de los usuarios, técnicos e industriales de la edificación hacia los procedimientos y sistemas en que se basa el aprovechamiento de otras fuentes alternativas de energía, principalmente la solar. Esto ha generado un rápido desarrollo industrial y comercial que, en opinión de los autores de este libro, arrastran los siguientes defectos: un mimético tecnologismo respecto de los sistemas convencionales que violenta las peculiaridades de la energia solar (baja densidad y variabilidad en el tiempol, y una escasa selectividad en la aplicación de los sistemas y procedimientos pasivos dando origen a un ecumenismo arquitectónico solar, al margen de las condiciones climáticas y funcionales específicas de cada caso y lugar.

En este libro, utilizando criterios y metodología pedagógicos, se dan los fundamentos e instrumentos teórico-prácticos necesarios para el planteamiento de todo proyecto arquitectónico solar pasivo, de acuerdo con los principios éticos y económicos de conservación y ahorro de energía. Es decir: respeto de los presupuestos bioclimáticos, búsqueda de la máxima captación y acumulación de la radiación solar, y esmero en el aislamiento térmico de los cerramientos.

Un volumen encuadernado en cartulina ibiza plastificada, a cinco colores, de $16 \times 23 \mathrm{~cm}$, compuesto de 216 páginas, 217 figuras, 87 gráficos, 19 tablas y 10 cuadros. 